\title{
Hyperpigmented Upper Eyelid: A Clue to the Diagnosis of Facial Lichen Planus Pigmentosus in a Patient with Frontal Fibrosing Alopecia
}

\author{
Maria Fernanda Reis Gavazzoni Dias ${ }^{a}$ Hudson Dutra Rezende ${ }^{b}$ \\ Amanda Lofeu Cury ${ }^{a}$ Ralph M. Trüeb ${ }^{c}$ Enoï Vilar ${ }^{a}$ \\ a Universidade Federal Fluminense, Niterói, Brazil; ${ }^{b}$ Faculdade de Medicina de Campos Hospital Escola Álvaro Alvim, \\ Campos dos Goytacazes, Brazil; ' Center for Dermatology and Hair Diseases Professor Trüeb and University of Zurich, \\ Zurich, Switzerland
}

\section{Established Facts}

- The coexistence of lichen planus pigmentosus (LPPig) and frontal fibrosing alopecia (FFA) is well established in the literature.

- Typical dermoscopic features of LPPig have been reported to help differentiate it from other facial hyperpigmented disorders.

- A facial skin biopsy may be necessary to differentiate LPPig from other hyperpigmented dermatoses.

\section{Novel Insights}

- LPPig can affect the upper eyelid.

- The hyperpigmentation of the upper eyelid with typical dermoscopy of LPPig may be a clue to the diagnosis of FFA.

- The hyperpigmentation of the upper eyelid is a potential noninvasive tool for the diagnosis of facial LPPig.

\section{Keywords}

Alopecia · Dermoscopy · Frontal fribrosing alopecia ·

Hyperpigmentation - Lichen planus pigmentosus .

Melasma $\cdot$ Upper eyelid

\section{Abstract}

Facial lichen planus pigmentosus (LPPig), a rare variant of classic lichen planus, was first described in patients with frontal fibrosing alopecia (FFA) by Dlova [Br J Dermatol 2013; 168:439-442] in 2013. The diagnosis of facial LPPig is some- times not easy, since clinical signs and histopathological features may frequently be confused with melasma or postinflammatory hyperpigmentation. We describe a case of a postmenopausal black woman diagnosed with FFA who presented with an identical brown-grayish pigmentation of the face and upper eyelids and typical dermoscopy analysis on both regions. We suggest that the hyperpigmentation of the upper eyelid with typical LLPig dermoscopy (upper eyelid sign) may be a clue for the diagnosis of LPPig and may avoid a scar-causing face biopsy.

(c) 2018 S. Karger AG, Basel

\section{KARGER}

(c) 2018 S. Karger AG, Basel

E-Mail karger@karger.com

www.karger.com/sad
Maria Fernanda Reis Gavazzoni Dias, MD, PhD

Rua Mariz e Barros, 176/607-608 Icaraí

Niterói, RJ 24220-121 (Brazil)

E-Mailmgavazzoni@gmail.com 


\section{Introduction}

First described in 1974 by Bhutani et al. [1], in Indian patients, lichen planus pigmentosus (LPPig) is considered a rare variant of classic lichen planus [2,3]. It most commonly affects female individuals with high skin phototypes (Fitzpatrick skin phototypes III to VI) and it is rarely seen in fair-skinned people $[3,4]$. In this work, we describe a postmenopausal female patient with late-stage frontal fibrosing alopecia (FFA) and facial LPPig involv- ing the upper eyelids. We call attention of the involvement of the upper eyelids as a possible clue to the differential diagnosis of LPPig and melasma.

\section{Case Report}

We describe the case of a 56-year-old postmenopausal black woman complaining of hair loss and face darkening during the last 4 years. She had been diagnosed as having melasma and had used
Fig. 1. a Important cicatricial alopecia affecting the eyebrows and scalp, except for the occipital area. b Intense reticular brown-grayish hyperpigmentation involving the face and upper eyelids.

Fig. 2. Dermoscopy of the facial hyperpigmentation (a) and upper eyelid (b) with a hand-held dermoscope (DermLite DL4 ${ }^{\circledR}$, $\times 20$ magnification). Blue-grey dots (brackets) and brown globules were accompanied by an exaggerated pseudoreticular pigmentary network pattern (asterisks). There is a blue-grey pigmentation around asymmetrically distributed follicular openings (black arrows). b The white arrows show rhomboidal structures, and diffuse erythema is noted. A few vellus hairs are observed in both images.
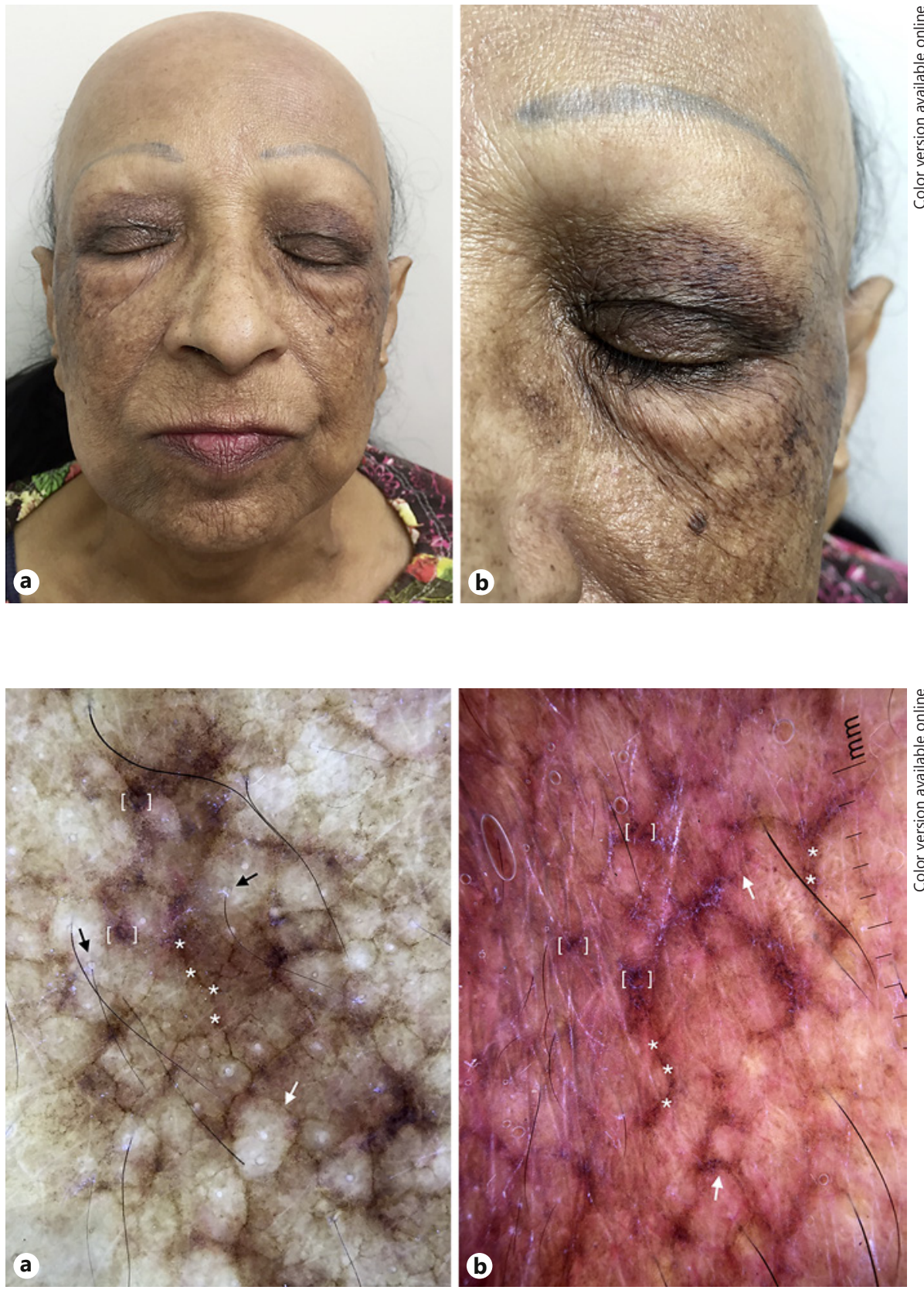
hydroquinone creams in the past, with no response. She was otherwise healthy.

On physical examination, she presented a severe recession of the frontotemporal hairline, parietal and temporal scalp alopecia, and absence of sideburns and eyebrows. Malar regions were hyperpigmented with a peculiar brown-grayish color, and the upper eyelids displayed an even more noticeable hyperpigmentation (Fig. 1), which strongly resembled the facial pattern, both clinically and dermoscopically (Fig. 2). Dermoscopy interpretation and description were based on studies conducted by Sharma et al. [2] and Pirmez et al. [5].

A skin biopsy of the scalp evidenced perifollicular lymphocytic inflammatory infiltrate, concentric lamellar fibrosis, and a lichenoid folliculitis. One dermoscopy-guided punch biopsy was obtained from the left malar region. The histopathology showed discrete lichenoid interface dermatitis with pigment incontinence, numerous dermal melanophages, and focal vacuolar degeneration of the basal cell layer (Fig. 3). Correlation of clinical, histopathological and dermoscopic features confirmed the diagnosis of FFA combined with facial and upper eyelid LPPig.

\section{Discussion}

The coexistence of LPPig and FFA was first described by Dlova [6] in 2013 in 24 South African patients who lately developed FFA. Since then, the association with other comorbidities such as other variants of LP, endocrinopathies, and lupus erythematosus has been reported by other authors $[3,7]$, with a possible genetic background involving the pathogenesis of FFA [8].

The diagnosis of facial LPPig is sometimes not easy since clinical signs and histopathological features may

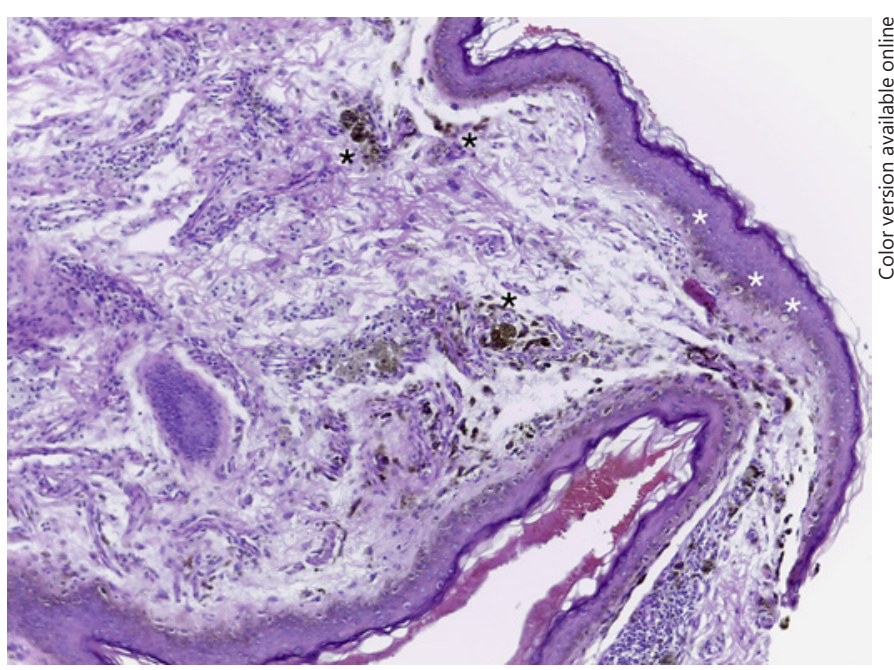

Fig. 3. Focal vacuolar degeneration of the basal cell layer (white asterisks) and pigment incontinence (black asterisks). H\&E. $\times 40$. frequently be confused with melasma or postinflammatory hyperpigmentation, and a thorough dermoscopy analysis may thus play an important role in distinguishing such entities.

The finding of a classic LPPig on the upper eyelid, sharing typical LPPig dermoscopy features $[2,5]$ and characteristic histopathological findings [9-11], may call attention to the fact that the eyelid involvement may not be anecdotal. Instead, it may be an important clue to the diagnosis of LPPig that helps differentiating it from other common photodermatoses such as melasma because the upper eyelid is a well-known sun-protected area. A face biopsy is not always possible to perform, as pointed out in the work of Pirmez et al. [5], and in late stages, the characteristic lichenoid dermatitis may already have disappeared.

Although the real frequency of the upper eyelid involvement in facial LPPig is unknown, we suggest that, when present, it may be considered a clue to the diagnosis of LPPig in patients with primary cicatricial alopecias of the lichen planopilaris group and may well replace the need for a scar-causing face biopsy [12]. It is possible that the involvement of the upper eyelid is a fortuitous feature, and the study of a more significant number of patients is needed to establish the actual significance of this association.

\section{Statement of Ethics}

The authors of this article state that the patient gave her informed consent and that the study protocol was approved by the Ethics Committee for Human Research of Universidade Federal Fluminense.

\section{Disclosure Statement}

The authors declare no conflicts of interest.

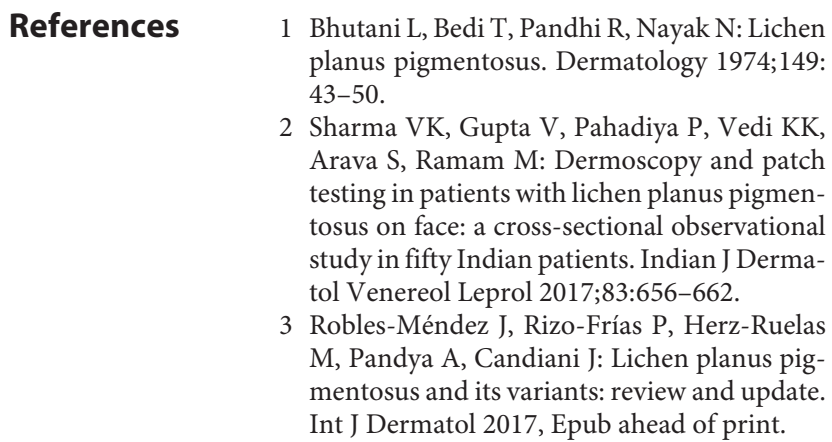


4 Lemes L, Verde R, Durães S, Araripe A, Pantaleão L: Coexistence of nail lichen planus and lichen planus pigmentosus. An Bras Dermatol 2016;91:20-22.

5 Pirmez R, Duque-Estrada B, Donati A, Campos-do-Carmo G, Valente NS, Romiti R, Sodré CT, Tosti A: Clinical and dermoscopic features of lichen planus pigmentosus in 37 patients with frontal fibrosing alopecia. $\mathrm{Br} \mathrm{J}$ Dermatol 2016;175:1387-1390.

6 Dlova NC: Frontal fibrosing alopecia and lichen planus pigmentosus: is there a link? $\mathrm{Br} J$ Dermatol 2013;168:439-442.
7 Trüeb R, Shabrawi-Caelen L, Kempf W: Cutaneous lupus erythematosus presenting as frontal fibrosing alopecia: report of 2 patients. Skin Appendage Disord 2017;3:205-210.

$\checkmark 8$ Missio D, Gavazzoni MF, Trüeb R: Familial cicatricial alopecia: report of familial frontal fibrosing alopecia and fibrosing alopecia in a pattern distribution. Int J Trichology 2017;9: 130-134.

9 Hsu CY, Liu D, Lee WR, Shih YH: Lichen planus pigmentosus inversus caused by occupational systemic sensitization to metals in a semiconductor factory worker. Dermatitis 2017;28:324-326.
10 Kanwar AJ, Dogra S, Handa S, Parsad BD, Radotrat B: A study of 124 Indian patients with lichen planus pigmentosus. Clin Exp Dermatol 2003;28:481-485.

11 Vega ME, Waxtein L, Arenas R, Hojyo T, Dominguez-Soto L: Ashy dermatosis and lichen planus pigmentosus: a clinicopathologic study of 31 cases. Int J Dermatol 1992;31:9094.

2 Abhishek K, Khunger N: Complications of skin biopsy. J Cutan Aesthet Surg 2015;8:239241 